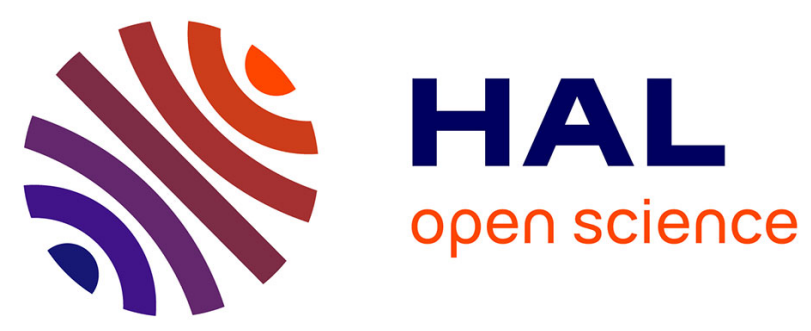

\title{
Renewable polymers derived from ferulic acid and biobased diols via ADMET
}

Imane Barbara, Amandine Flourat, Florent Allais

\section{To cite this version:}

Imane Barbara, Amandine Flourat, Florent Allais. Renewable polymers derived from ferulic acid and biobased diols via ADMET. European Polymer Journal, 2015, 62, pp.236-243. 10.1016/j.eurpolymj.2014.11.035 . hal-01598634

HAL Id: hal-01598634

https://hal-agroparistech.archives-ouvertes.fr/hal-01598634

Submitted on 8 Feb 2019

HAL is a multi-disciplinary open access archive for the deposit and dissemination of scientific research documents, whether they are published or not. The documents may come from teaching and research institutions in France or abroad, or from public or private research centers.
L'archive ouverte pluridisciplinaire HAL, est destinée au dépôt et à la diffusion de documents scientifiques de niveau recherche, publiés ou non, émanant des établissements d'enseignement et de recherche français ou étrangers, des laboratoires publics ou privés. 


\title{
Renewable polymers derived from ferulic acid and biobased diols via ADMET
}

\author{
Imane Barbara ${ }^{a}$, Amandine L. Flourat ${ }^{\mathrm{a}, \mathrm{b}, \mathrm{c}}$, Florent Allais ${ }^{\mathrm{a}, \mathrm{d}, \mathrm{e} *}$

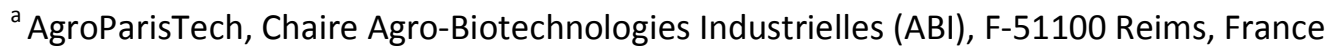 \\ ${ }^{\mathrm{b}}$ AgroParisTech, UMR 1318 IJPB, F-78026 Versailles, France \\ ' INRA, UMR 1318 IJPB, F-78026 Versailles, France \\ ${ }^{d}$ AgroParisTech, UMR 782 GMPA, F-78850 Thiverval-Grignon, France \\ e INRA, UMR 782 GMPA, F-78850 Thiverval-Grignon, France
}

Correspondence to: Prof. F. Allais (E-mail: florent.allais@agroparistech.fr)

Additional Supporting Information may be found in the online version of this article.

\begin{abstract}
Renewable $\alpha, \omega$-diene monomers have been prepared from ferulic acid, biosourced diols (isosorbide and butanediol) and bromo-alkenes using a chemo-enzymatic synthetic pathway then studied as monomers in ADMET polymerization. All monomers and polymers have been thoroughly characterized using NMR, GPC, DSC and TGA. ADMET polymerization was optimized with regard to the catalyst loading (Hoveyda-Grubbs II), the reaction medium (in mass vs. in solvent), and the temperature, and led to polymers with molecular weight up to $25 \mathrm{kDa}$. Thermal analysis of these new poly(ester-alkenamer)s showed excellent thermal stabilities $\left(283-370{ }^{\circ} \mathrm{C}\right.$ ) and tunable $T_{g}$ depending on the nature of the biobased diol and the chain length of the alkene in the $\alpha, \omega$-diene monomer.
\end{abstract}

KEYWORDS: polyesters, ADMET polymerization, $\alpha, \omega$-dienes, ferulic acid, bisphenol, lignocellulose

\section{INTRODUCTION}

The collapse of fossil resources and rising prices may have been the first trigger for a biobased economy, but today, industries, customers and regulators (e.g., $\mathrm{REACH}$ ) are increasingly demanding for eco-friendly and bio-based chemicals, as well as sustainable industrial processes. In this context, the production of new platform chemicals from biomass through green processes is an alluring strategy for a sustainable development. Indeed, biomass offers a wide range of molecules that can be used to access valuable synthons such as polyols, furans, fatty acids, aliphatic alkanes/alkenes or aromatics/phenolics to name a few. ${ }^{1}$ Ferulic acid (1), one of the three $p$-hydroxycinnamic acids found in lignocellulosic biomass is one of these sustainable and valuable chemical feedstocks. Present in relatively large quantities in wheat and rice brans as well as in sugarbeet pulp or sugarcane bagasse, its production at industrial scale from these biorefineries and food industries byproducts is been investigated, particularly in our team. According to the best estimates, prices could be as low as \$1-3 per $\mathrm{kg}$, which puts it at a reasonable price for a monomer. It is noteworthy that ferulic acid can also be readily synthesized from vanillin, a phenolic compound industrially produced from lignins (sale price ca. \$6-15 per kg), however this synthetic pathway requires extra synthetic/purifications step leading to higher production cost. 
The $\alpha, \beta$-unsaturated carboxylic acid and phenol functional groups present in ferulic acid offer a wide range of possibilities in terms of (bio)chemical transformations, and opens the way for the synthesis of value-added molecules such as drugs, functional additives (e.g., antioxidants), monomers and polymers. Many research groups have recently demonstrated the great potential of ferulic acid-derived ABtype monomers through the development of new homopolyesters exhibiting appealing thermal properties through either metalcatalyzed polycondensation, ${ }^{2}$ oxalyl- ${ }^{3}$ or thionylchloride- ${ }^{4}$ activated polycondensations. Other homo- and copolyesters of natural $p$ hydroxycinnamic acids showing remarkable liquid-crystal, ${ }^{5}$ biodegradability ${ }^{6}$ or biocompatibility $^{5 b}$ properties were also reported.

Having developed expertise in the chemistry of lignin related phenolic compounds, ${ }^{7}$ we recently contributed to this research topic through the synthesis of a new class of bio-based polyfunctional molecules derived from ferulic acid. We indeed designed a new methodology for the production of bisphenols incorporating ferulic acid and bio-based diols through a chemo-enzymatic process involving Candida antarctica lipase B laka CAL-B, Novozyme $\left.{ }^{\circledR} 435\right){ }^{8}$ This strategy allowed the incorporation of aromatic and aliphatic segments into renewable $A_{2}$-type bisphenolic structures under mild conditions in bulk as well as in solvent. Recently, these bisphenolic structures were efficiently condensed with renewable activated diacid chlorides (i.e., succinoyl and azelaoyl acid chlorides) and two isocyanates thereby providing renewable alternating aliphatic-aromatic copolyesters ${ }^{9}$ and poly(ester-urethane)s ${ }^{10}$, respectively, whose thermal properties could be finely tuned by judiciously playing with the chemical structure of both the diacid, or diisocyanate, and the bisphenol.
Thanks to the ease of handling and high functional group tolerance of the Ru-based catalysts used in acyclic diene metathesis (ADMET), this method is a very useful approach for the construction of defined polymer architectures $^{11}$ and allowed the synthesis of renewable polymers, such as polyesters, polyethers, polyamides and many others, very promising for commercial applications. ${ }^{12}$ Even though extensive research has been performed to develop commercial ferulic acid derived renewable polymers, ${ }^{2,8-10}$ to the best of our knowledge, there is only one example of ADMET polymerization involving ferulic acid derivatives as diene substrates. Indeed, Meier and co-workers reported on the use of a bisallylated ferulic acid derivative (2) as $\alpha, \omega$ diene monomer for the synthesis of renewable poly(ester-alkenamer)s (Scheme 1). ${ }^{12 a}$ In their study, homopolymerization and copolymerization of $\mathbf{2}$ with oleic and erucicderived $\alpha, \omega$-dienes resulted in polymers with molecular weights of 2-3 KDa and $9 \mathrm{kDa}$, respectively. Though these polymers were obtained in relatively low molecular weights, this study demonstrated the potentialities of

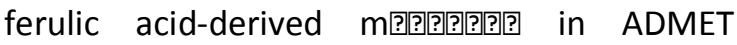
chemistry.

In this work, the ferulic acid-based bisphenols, obtained through lipase-mediated chemoenzymatic synthesis and described above, have been used as precursors for the synthesis of novel $\alpha, \omega$-dienes monomers incorporating dihydroferulate ester moieties. These monomers were then submitted to ADMET polymerization to determine their suitability to such method and compare their reactivity to previously reported ferulic-acid derived $\alpha, \omega$-diene monomer $\mathbf{2}$. The structure and thermal properties of the novel poly(esteralkenamer)s were then studied in order to evaluate their potential in industrial applications. 


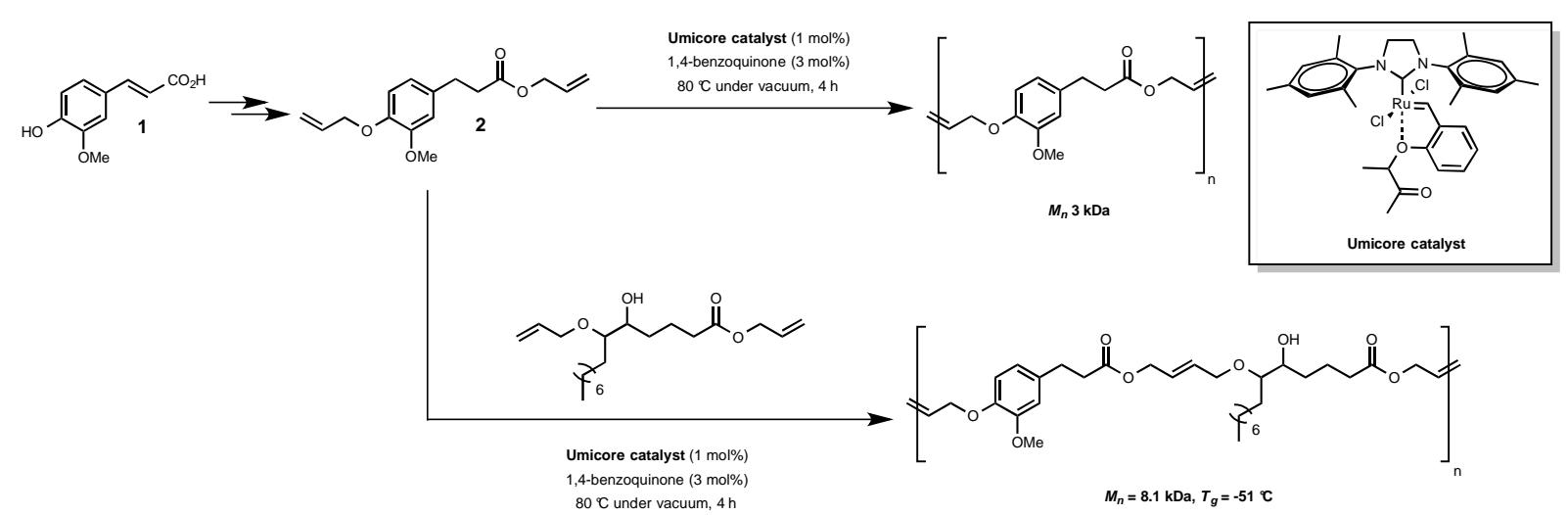

Scheme 1. Synthesis of the first ferulic acid-derived ADMET polymers by Meier and co-workers ${ }^{12 a}$

\section{EXPERIMENTAL}

\section{Materials and methods}

All reagents were purchased from Sigma-Aldrich and used as received. Solvents were purchased from ThermoFisher Scientific, DMF was dried on a mBraun SPS 800 system. Deuterated chloroform $\left(\mathrm{CDCl}_{3}\right)$ was purchased from Eurisotop. Evaporations were conducted under reduced pressure at temperature below $40{ }^{\circ} \mathrm{C}$. Column chromatography was carried out with an automated flash chromatography (PuriFlash 4100, Interchim) and pre-packed INTERCHIM PF-30SI-HP (30 $\mu \mathrm{m}$ silica gel) columns. FT-IR and UV analyses were performed respectively on Cary 630 FTIR and Cary 60 UV-Vis from Agilent technologies. NMR analyses were recorded on a Bruker Fourier 300. ${ }^{1} \mathrm{H}$ NMR spectra of samples were recorded in $\mathrm{CDCl}_{3}$ at $300 \mathrm{MHz}$, chemicals shifts were reported in parts per million relative to the internal standard tetramethylsilane (TMS, $\delta=0.00 \mathrm{ppm}$ ). ${ }^{13} \mathrm{C} \mathrm{NMR}$ spectra of samples were recorded at $75 \mathrm{MHz}\left(\mathrm{CDCl}_{3}\right.$ residual signal at $\delta=77.16 \mathrm{ppm})$. Thermogravimetric analyses (TGA) were recorded on a Q500, from TA. Around $5 \mathrm{mg}$ of each sample was heated at $10{ }^{\circ} \mathrm{C} \cdot \mathrm{min}^{-1}$ from 25 to $500{ }^{\circ} \mathrm{C}$ under nitrogen flow (60 mL. $\left.\mathrm{min}^{-1}\right)$. Differential scanning calorimetry (DSC) thermograms were obtained using a DSC Q20, from TA, under inert atmosphere $\left(\mathrm{N}_{2}\right)$. Around $5 \mathrm{mg}$ were weighed in a pan which was then sealed and submitted to the following heat/cool/heat cycle: equilibration at $-75{ }^{\circ} \mathrm{C}$ and hold for $5 \mathrm{~min}$, heating from $-75^{\circ} \mathrm{C}$ to $150{ }^{\circ} \mathrm{C}$ at $10^{\circ} \mathrm{C} \cdot \mathrm{min}^{-1}$ and hold for $5 \mathrm{~min}$, cooling from $150{ }^{\circ} \mathrm{C}$ to $-75^{\circ} \mathrm{C}$ at $10{ }^{\circ} \mathrm{C} \cdot \mathrm{min}^{-1}$. Gel Permeation Chromatography (GPC) was performed at $40{ }^{\circ} \mathrm{C}$ on an Infinity 1260 system from Agilent Technologies with a quadruple detection (IR, UV, MALS, viscosimetry) and two PL-Gel $5 \mathrm{~mm}$ Mixed D column (300 mm $\times 7.5 \mathrm{~mm}$ ) in THF (flow rate 1 $\mathrm{mL} \cdot \mathrm{min}^{-1}$ ) using polystyrene calibration.

\section{General procedure for the synthesis of $\alpha, \omega$ - diene monomers}

Bisphenol ( 1 equiv) and $\mathrm{K}_{2} \mathrm{CO}_{3}$ ( 3 equiv) were dissolved in dry $\mathrm{DMF}(\mathrm{C}=2.5 \mathrm{M})$ under nitrogen. Bromo-alkene (2.5 equiv) was then added, and the mixture was stirred and heated at $80{ }^{\circ} \mathrm{C}$ for $12 \mathrm{~h}$. Reaction was quenched with water $\left(v\left(\mathrm{H}_{2} \mathrm{O}\right)=\mathrm{v}(\mathrm{DMF})\right)$ and the aqueous layer was extracted three times with ethyl acetate ( 3 $x \mathrm{v}\left(\mathrm{H}_{2} \mathrm{O}\right)$ ). Organic layers were combined, washed with brine, dried over anhydrous $\mathrm{MgSO}_{4}$, filtered and concentrated. Crude product was purified by flash chromatography 
on silica gel Cyclohexane:AcOEt 75:25 to yield the corresponding $\alpha, \omega$-diene monomer.

Bis-Allyl-O-Dihydroferuloyl 1,4-Butanediol (BDFAll)

White powder (81\%). UV: $\lambda_{\max }(\mathrm{EtOH}): 211,230$, $281 \mathrm{~nm}$. FTIR (neat): $v_{\max }: 1728(\mathrm{C}=0), 1510$ (C=C Ar). ${ }^{1} \mathrm{H}$ NMR $\left(300 \mathrm{MHz}, \mathrm{CDCl}_{3}, \delta\right): 6.72(2 \mathrm{H}$, $\left.m, H_{8}\right), 6.69\left(4 \mathrm{H}, \mathrm{m}, \mathrm{H}_{5,9}\right), 6.00-6.12\left(2 \mathrm{H}, \mathrm{m}, \mathrm{H}_{14}\right)$, 5.24-5.42 (4H, m, $\left.\mathrm{H}_{15}\right), 4.57(4 \mathrm{H}, \mathrm{dt}, J 5.4, J 1.5$, $\left.\mathrm{H}_{13}\right), 4.07\left(4 \mathrm{H}, \mathrm{m}, \mathrm{H}_{11}\right), 3.85\left(6 \mathrm{H}, \mathrm{s}, \mathrm{H}_{10}\right), 2.88$ $\left(4 \mathrm{H}, \mathrm{m}, \mathrm{H}_{3}\right), 2.60\left(4 \mathrm{H}, \mathrm{m}, \mathrm{H}_{2}\right), 1.64\left(4 \mathrm{H}, \mathrm{m}, \mathrm{H}_{12}\right)$. ${ }^{13} \mathrm{C}\left(75 \mathrm{MHz}, \mathrm{CDCl}_{3}, \delta\right): 172.9\left(\mathrm{C}_{1}\right), 149.3\left(\mathrm{C}_{6}\right)$, $146.4\left(C_{7}\right), 133.5\left(C_{4,14}\right), 120.0\left(C_{9}\right), 117.8\left(C_{15}\right)$, $113.5\left(C_{8}\right), 112.0\left(C_{5}\right), 70.0\left(C_{13}\right), 63.8\left(C_{11}\right), 55.8$ $\left(C_{10}\right), 36.0\left(C_{2}\right), 30.6\left(C_{3}\right), 25.2\left(C_{12}\right)$

Bis-Hexene-O-Dihydroferuloyl 1,4-Butanediol (BDF-Hex)

White powder (80\%). UV: $\lambda_{\max }(\mathrm{EtOH}): 235,281$ nm. FTIR (neat): $v_{\text {max }}: 1729(\mathrm{C}=0), 1512$ ( $C=C$ Ar). ${ }^{1} \mathrm{H}$ NMR $\left(300 \mathrm{MHz}, \mathrm{CDCl}_{3}, \delta\right): 6.78\left(2 \mathrm{H}, \mathrm{m}, \mathrm{H}_{8}\right)$, $6.69\left(4 \mathrm{H}, \mathrm{m}, \mathrm{H}_{5,9}\right), 5.75-5.86\left(2 \mathrm{H}, \mathrm{m}, \mathrm{H}_{17}\right)$, 4.94$5.05\left(4 \mathrm{H}, \mathrm{m}, \mathrm{H}_{18}\right), 4.07\left(4 \mathrm{H}, \mathrm{m}, \mathrm{H}_{11}\right), 3.98(4 \mathrm{H}, \mathrm{t}, \mathrm{J}$ 6.6, $\left.\mathrm{H}_{13}\right), 3.84\left(6 \mathrm{H}, \mathrm{s}, \mathrm{H}_{10}\right), 2.88\left(4 \mathrm{H}, \mathrm{m}, \mathrm{H}_{3}\right), 2.60$ $\left(4 \mathrm{H}, \mathrm{m}, \mathrm{H}_{2}\right), 2.12\left(4 \mathrm{H}, \mathrm{m}, \mathrm{H}_{16}\right), 1.86\left(4 \mathrm{H}, \mathrm{m}, \mathrm{H}_{14}\right)$, $1.78\left(4 \mathrm{H}, \mathrm{m}, \mathrm{H}_{12}\right) .{ }^{13} \mathrm{C}\left(75 \mathrm{MHz}, \mathrm{CDCl}_{3}, \delta\right): 172.9$ $\left(C_{1}\right), 149.3\left(C_{6}\right), 147.0\left(C_{7}\right), 138.5\left(C_{17}\right), 133.1$ $\left(C_{4}\right), 120.1\left(C_{9}\right), 114.7\left(C_{18}\right), 113.0\left(C_{8}\right), 112.0$ $\left(C_{5}\right), 68.8\left(C_{13}\right), 63.8\left(C_{11}\right), 55.9\left(C_{10}\right), 36.1\left(C_{2}\right)$, $33.4\left(C_{16}\right), 30.6\left(C_{3}\right), 28.6\left(C_{14}\right), 25.2\left(C_{12,15}\right)$.

Bis-Decene-O-Dihydroferuloyl 1,4-Butanediol (BDF-Dec)

Colorless oil (82\%). UV: $\lambda_{\max }$ (EtOH): 228, 281 nm. FTIR (neat): $v_{\max }: 1732(\mathrm{C}=0), 1512(\mathrm{C}=\mathrm{C}$ Ar). ${ }^{1} \mathrm{H} \mathrm{NMR}\left(300 \mathrm{MHz}, \mathrm{CDCl}_{3}, \delta\right): 6.77(2 \mathrm{H}, \mathrm{m}$, $\left.\mathrm{H}_{8}\right), 6.69\left(4 \mathrm{H}, \mathrm{m}, \mathrm{H}_{5,9}\right), 5.73-5.87\left(2 \mathrm{H}, \mathrm{m}, \mathrm{H}_{21}\right)$, 4.90-5.02 (4H, m, $\left.\mathrm{H}_{22}\right), 4.09\left(4 \mathrm{H}, \mathrm{m}, \mathrm{H}_{11}\right), 3.96$ $\left(4 \mathrm{H}, \mathrm{t}, J\right.$ 6.9, $\left.\mathrm{H}_{13}\right), 3.84\left(6 \mathrm{H}, \mathrm{s}, \mathrm{H}_{10}\right), 2.88(4 \mathrm{H}, \mathrm{m}$, $\left.\mathrm{H}_{3}\right), 2.60\left(4 \mathrm{H}, \mathrm{m}, \mathrm{H}_{2}\right), 2.05\left(4 \mathrm{H}, \mathrm{m}, \mathrm{H}_{20}\right), 1.79(4 \mathrm{H}$, $\left.m, \mathrm{H}_{14}\right), 1.64\left(4 \mathrm{H}, \mathrm{m}, \mathrm{H}_{12}\right), 1.31-1.42(2 \mathrm{OH}, \mathrm{m}$, $\left.\mathrm{H}_{15-19}\right) .{ }^{13} \mathrm{C}\left(75 \mathrm{MHz}, \mathrm{CDCl}_{3}, \delta\right): 172.9\left(\mathrm{C}_{1}\right), 149.3$ $\left(C_{6}\right), 147.0\left(C_{7}\right), 139.1\left(C_{21}\right), 133.0\left(C_{4}\right), 120.1$ $\left(C_{9}\right), 114.1\left(C_{22}\right), 113.0\left(C_{8}\right), 112.0\left(C_{5}\right), 69.1\left(C_{13}\right)$, $63.8\left(C_{11}\right), 55.9\left(C_{10}\right), 36.1(C), 33.8\left(C_{20}\right), 30.6$ $\left(C_{3}\right), 25.9-29.4\left(C_{15-19}\right), 25.2\left(C_{12}\right)$.

Bis-allyl-O-Dihydroferuloyl Isosorbide (IDF-AII)

White powder (60\%) UV: $\lambda_{\max }(\mathrm{EtOH}): 205,228$, $281 \mathrm{~nm}$. FTIR (neat): $v_{\max }: 1719$ (C=O), 1513 (C=C Ar) $\mathrm{cm}^{-1} .{ }^{1} \mathrm{H}$ NMR $\left(300 \mathrm{MHz}, \mathrm{CDCl}_{3}, \delta\right): 6.55-6.72$

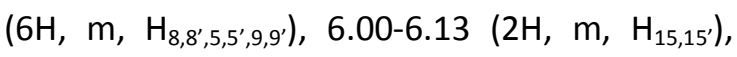
5.20-5.50 (4H, m, $\left.\mathrm{H}_{16,16^{\prime}}\right), 5.17\left(1 \mathrm{H}, \mathrm{m}, \mathrm{H}_{11^{\prime}}\right), 5.12$ $\left(1 \mathrm{H}, \mathrm{m}, \mathrm{H}_{11}\right), 4.77\left(1 \mathrm{H}, \mathrm{t}, J\right.$ 4.8, $\left.\mathrm{H}_{13}\right), 4.57(4 \mathrm{H}, \mathrm{m}$, $\left.\mathrm{H}_{14,14^{\prime}}\right), 4.37\left(1 \mathrm{H}, \mathrm{d}, J 4.8, \mathrm{H}_{13}\right), 3.85(6 \mathrm{H}, \mathrm{s}$, $\left.\mathrm{H}_{10,10^{\prime}}\right), 3.71-3.93\left(4 \mathrm{H}, \mathrm{m}, \mathrm{H}_{12,12^{\prime}}\right), 2.89(4 \mathrm{H}, \mathrm{m}$, $\left.\mathrm{H}_{3,3^{\prime}}\right), 2.69\left(4 \mathrm{H}, \mathrm{m}, \mathrm{H}_{2,2^{\prime}}\right) \cdot{ }^{13} \mathrm{C}\left(75 \mathrm{MHz}, \mathrm{CDCl}_{3}, \delta\right)$ : $172.2\left(C_{1^{\prime}}\right), 171.8\left(C_{1}\right), 149.5\left(C_{6,6^{\prime}}\right), 146.6\left(C_{7,7}\right)$, 133.1-133.6 $\left(C_{\left.4^{\prime}, 4,15,15^{\prime}\right),} 120.1 \quad\left(C_{9,9^{\prime}}\right), \quad 117.7\right.$ $\left(C_{16,16^{\prime}}\right), 113.9\left(C_{8,8^{\prime}}\right), 112.2\left(C_{5,5^{\prime}}\right), 85.9\left(C_{13^{\prime}}\right), 80.7$ $\left(C_{13}\right), 78.0\left(C_{11^{\prime}}\right), 73.9\left(C_{11}\right), 70.3\left(C_{12,12^{\prime}}\right), 70.1$ $\left(C_{14,14^{\prime}}\right), 55.9\left(C_{10,10^{\prime}}\right), 35.7-35.8\left(C_{2,2^{\prime}}\right), 30.4\left(C_{3,3^{\prime}}\right)$.

Bis-Hexene-O-Dihydroferuloyl Isosorbide (IDFHex)

Viscous oil (78\%) UV: $\lambda_{\max }$ (EtOH): 202, 205, 228, $281 \mathrm{~nm}$. FTIR (neat): $v_{\max }: 1736(\mathrm{C}=0), 1511$ ( $\mathrm{C}=\mathrm{C}$ Ar) $\mathrm{cm}^{-1} .{ }^{1} \mathrm{H}$ NMR $\left(300 \mathrm{MHz}, \mathrm{CDCl}_{3}, \delta\right): 6.79$ $\left(2 \mathrm{H}, \mathrm{m}, \mathrm{H}_{8,8^{\prime}}\right), 6.72\left(4 \mathrm{H}, \mathrm{m}, \mathrm{H}_{5,5^{\prime}, 9,9^{\prime}}\right), 5.77-5.87$ $\left(2 \mathrm{H}, \mathrm{m}, \mathrm{H}_{18,18^{\prime}}\right), 5.18\left(1 \mathrm{H}, \mathrm{m}, \mathrm{H}_{11^{\prime}}\right), 5.12(1 \mathrm{H}, \mathrm{m}$, $\left.\mathrm{H}_{11}\right)$, 4.93-5.04 (4H, $\left.\mathrm{m}, \mathrm{H}_{19,19^{\prime}}\right), 4.78(1 \mathrm{H}, \mathrm{t}, J$ J 4.9, $\left.\mathrm{H}_{13^{\prime}}\right), 4.38\left(1 \mathrm{H}, \mathrm{d}, J 4.8, \mathrm{H}_{13}\right), 3.98-4.00(4 \mathrm{H}, \mathrm{m}$, $\left.\mathrm{H}_{14,14^{4}}\right), 3.91\left(6 \mathrm{H}, \mathrm{s}, \mathrm{H}_{10,10^{\prime}}\right), 3.76-3.93(4 \mathrm{H}, \mathrm{m}$, $\left.\mathrm{H}_{12,12^{\prime}}\right), 2.87\left(4 \mathrm{H}, \mathrm{m}, \mathrm{H}_{3,3^{\prime}}\right), 2.65\left(4 \mathrm{H}, \mathrm{m}, \mathrm{H}_{2,2^{\prime}}\right)$, $2.12\left(4 \mathrm{H}, \mathrm{m}, \mathrm{H}_{17,17^{\prime}}\right), 1.82\left(4 \mathrm{H}, \mathrm{m}, \mathrm{H}_{15,15^{\prime}}\right), 1.57$ $\left(4 \mathrm{H}, \mathrm{m}, \mathrm{H}_{16,16^{\prime}}\right) \cdot{ }^{13} \mathrm{C}\left(75 \mathrm{MHz}, \mathrm{CDCl}_{3}, \delta\right): 172.3$ $\left(C_{1^{\prime}}\right), 172.0\left(C_{1}\right), 149.3\left(C_{6,6^{\prime}}\right), 147.0\left(C_{7,7^{\prime}}\right), 138.6$ $\left(C_{18,18^{\prime}}\right), 132.9\left(C_{4}\right), 132.7\left(C_{4^{\prime}}\right), 120.1\left(C_{9,9^{\prime}}\right), 114.7$ $\left(C_{19,19^{\prime}}\right), 113.1\left(C_{8,8^{\prime}}\right), 112.1\left(C_{5,5^{\prime}}\right), 85.9\left(C_{13^{\prime}}\right), 80.7$ $\left(C_{13}\right), 77.9\left(C_{11^{\prime}}\right), 73.9\left(C_{11}\right), 73.4\left(C_{12^{\prime}}\right), 70.3\left(C_{12}\right)$, $68.9\left(C_{14,14^{\prime}}\right), 56.0\left(C_{10,10^{\prime}}\right), 36.0\left(C_{2^{\prime}}\right), 35.7\left(C_{2}\right)$, 
$33.4\left(C_{17,17^{\prime}}\right), 30.4\left(C_{3,3^{\prime}}\right), 28.6\left(C_{15,15^{\prime}}\right), 25.2$ $\left(\mathrm{C}_{16,16^{6}}\right)$.

Bis-Decene- O-Dihydroferuloyl Isosorbide (IDFDec)

Colorless oil (79\%) UV: $\lambda_{\max }(\mathrm{EtOH}): 204,228$, $281 \mathrm{~nm}$. FTIR (neat): $v_{\max }: 1722(\mathrm{C}=0), 1516$ (C=C Ar) $\mathrm{cm}^{-1} \cdot{ }^{1} \mathrm{H}$ NMR $\left(300 \mathrm{MHz}, \mathrm{CDCl}_{3}, \delta\right): 6.79$ $\left(2 \mathrm{H}, \mathrm{m}, \mathrm{H}_{8,8^{\prime}}\right), 6.70-6.72\left(4 \mathrm{H}, \mathrm{m}, \mathrm{H}_{5,5^{\prime}, 9,9^{\prime}}\right), 5.76-$ $5.85\left(2 \mathrm{H}, \mathrm{m}, \mathrm{H}_{22,22^{\prime}}\right), 5.17\left(1 \mathrm{H}, \mathrm{m}, \mathrm{H}_{11^{\prime}}\right), 5.13(1 \mathrm{H}$, $\left.\mathrm{m}, \mathrm{H}_{11}\right), 4.90-5.02\left(4 \mathrm{H}, \mathrm{m}, \mathrm{H}_{23,23^{\prime}}\right), 4.77(1 \mathrm{H}, \mathrm{m}$, $\left.\mathrm{H}_{13^{\prime}}\right), 4.38\left(1 \mathrm{H}, \mathrm{m}, \mathrm{H}_{13}\right), 3.98\left(4 \mathrm{H}, \mathrm{m}, \mathrm{H}_{14,14^{\prime}}\right), 3.91$ $\left(6 \mathrm{H}, \mathrm{s}, \mathrm{H}_{10,10^{\circ}}\right), 3.76-3.95\left(4 \mathrm{H}, \mathrm{m}, \mathrm{H}_{12,12^{\prime}}\right), 2.89(4 \mathrm{H}$, $\left.\mathrm{m}, \mathrm{H}_{3,3^{\prime}}\right), 2.65\left(4 \mathrm{H}, \mathrm{m}, \mathrm{H}_{2,2^{\prime}}\right), 2.03\left(4 \mathrm{H}, \mathrm{m}, \mathrm{H}_{15,15^{\prime}}\right)$, $1.82\left(4 \mathrm{H}, \mathrm{m}, \mathrm{H}_{17,17^{\prime}}\right), 1.31-1.50(2 \mathrm{H}, \mathrm{m}$,

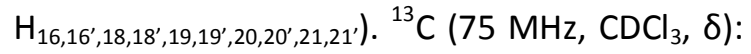
$172.3\left(C_{1^{\prime}}\right), 171.9\left(C_{1}\right), 149.3\left(C_{6,6^{\prime}}\right), 147.0\left(C_{7,7}\right)$, $139.2\left(C_{22,22^{\prime}}\right), 132.8\left(C_{4}\right), 132.6\left(C_{4^{\prime}}\right), 120.1\left(C_{9,9^{\prime}}\right)$, $114.2\left(C_{23,23^{\prime}}\right), 113.0\left(C_{8,8^{\prime}}\right), 112.10\left(C_{5,5^{\prime}}\right), 85.8$ $\left(\mathrm{C}_{13^{\prime}}\right), 80.7\left(\mathrm{C}_{13}\right), 77.9\left(\mathrm{C}_{11^{\prime}}\right), 73.9\left(\mathrm{C}_{11}\right), 73.3\left(\mathrm{C}_{12^{\prime}}\right)$, $70.3\left(C_{12}\right), 69.1\left(C_{14,14^{\prime}}\right), 55.9\left(C_{10,10^{\prime}}\right), 35.9\left(C_{2^{\prime}}\right)$, $35.7\left(C_{2}\right), 33.8\left(C_{21,21^{1}}\right), 30.4\left(C_{3,3^{3}}\right), \quad 28.9-29.4$

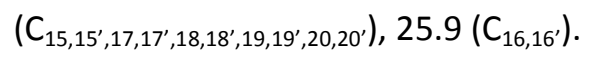

\section{General Procedure for ADMET polymerization}

\section{In mass polymerization}

Monomer ( 1 eq.) and $p$-benzoquinone ( 2 eq. relative to Hoveyda-Grubbs II catalyst) were stirred at $80{ }^{\circ} \mathrm{C}$, under vacuum for $5 \mathrm{~min}$. Hoveyda-Grubbs II catalyst (1-10 mol\%) was added and the reaction was continued, under vacuum, for $4 \mathrm{~h}$. Reaction was quenched with 1 $\mathrm{mL}$ of THF and 4 drops of vinyl ethyl ether. Product was concentrated and a sample was taken for GPC analyses. The remainder was dissolved in THF (1 $\mathrm{mL})$ and precipitated in cold methanol $(10 \mathrm{~mL})$.

\section{In solvent polymerization}

Monomer ( 1 eq.) and $p$-benzoquinone ( 2 eq. relative to Hoveyda-Grubbs II catalyst) were dissolvent in dry DCM (2 M). Hoveyda-Grubbs II catalyst (1-10 mol\%) was added and the reaction was continued, under nitrogen, at 40 ${ }^{\circ} \mathrm{C}$ for $4 \mathrm{~h}$. Reaction was quenched with $1 \mathrm{~mL}$ of THF and 4 drops of vinyl ethyl ether. Product was concentrated and a sample was taken for GPC analyses. The remainder was dissolved in THF $(1 \mathrm{~mL})$ and precipitated in cold methanol (10 mL).

P2 : $92 \%$ yield, ${ }^{1} \mathrm{H}$ NMR (300 $\mathrm{MHz}, \mathrm{CDCl}_{3}, \delta$ ): 6.68-6.80 (6H, m, $\left.\mathrm{H}_{5,8,9}\right), 5.43\left(2 \mathrm{H}, \mathrm{m}, \mathrm{H}_{17}\right), 4.08$ $\left(4 \mathrm{H}, \mathrm{m}, \mathrm{H}_{11}\right), 3.97\left(4 \mathrm{H}, \mathrm{t}, J 6.6, \mathrm{H}_{13}\right), 3.84(6 \mathrm{H}, \mathrm{s}$, $\left.\mathrm{H}_{10}\right), 2.88\left(4 \mathrm{H}, \mathrm{m}, \mathrm{H}_{3}\right), 2.60\left(4 \mathrm{H}, \mathrm{m}, \mathrm{H}_{2}\right), 1.30-$ $2.30\left(16 \mathrm{H}, \mathrm{m}, \mathrm{H}_{12,14,15,16}\right) .{ }^{13} \mathrm{C}\left(75 \mathrm{MHz}, \mathrm{CDCl}_{3}, \delta\right)$ : $173.1\left(C_{1}\right), 149.5\left(C_{6}\right), 147.2\left(C_{7}\right), 133.3\left(C_{4}\right)$, $130.4\left(C_{17}\right), 120.3\left(C_{9}\right), 113.2\left(C_{8}\right), 112.2\left(C_{5}\right)$, $69.1\left(C_{13}\right), 64.0\left(C_{11}\right), 56.1\left(C_{10}\right), 36.3\left(C_{2}\right), 32.5$ $\left(C_{16}\right), 30.7\left(C_{3}\right), 29.0\left(C_{14}\right), 25.4\left(C_{12,15}\right)$.

P3 : 90\% yield, ${ }^{1} \mathrm{H}$ NMR (300 $\mathrm{MHz}, \mathrm{CDCl}_{3}, \delta$ ): 6.69-6.80 (6H, m, $\left.\mathrm{H}_{5,8,9}\right), 5.38\left(2 \mathrm{H}, \mathrm{H}_{21}\right), 4.08(4 \mathrm{H}$, $\left.\mathrm{m}, \mathrm{H}_{11}\right), 3.96\left(4 \mathrm{H}, \mathrm{t}, J\right.$ 6.9, $\left.\mathrm{H}_{13}\right), 3.84\left(6 \mathrm{H}, \mathrm{s}, \mathrm{H}_{10}\right)$, $2.88\left(4 \mathrm{H}, \mathrm{m}, \mathrm{H}_{3}\right), 2.60\left(4 \mathrm{H}, \mathrm{m}, \mathrm{H}_{2}\right), 1.96(4 \mathrm{H}, \mathrm{m}$, $\left.\mathrm{H}_{20}\right), 1.81\left(4 \mathrm{H}, \mathrm{m}, \mathrm{H}_{14}\right), 1.65\left(4 \mathrm{H}, \mathrm{m}, \mathrm{H}_{12}\right), 1.21-$ $1.51\left(2 \mathrm{OH}, \mathrm{m}, \mathrm{H}_{15-19}\right) .{ }^{13} \mathrm{C}\left(75 \mathrm{MHz}, \mathrm{CDCl}_{3}, \delta\right)$ : $173.1\left(C_{1}\right), 149.3\left(C_{6}\right), 147.1\left(C_{7}\right), 133.1\left(C_{4}\right)$, $130.5\left(C_{21}\right), 120.2\left(C_{9}\right), 113.0\left(C_{8}\right), 112.1\left(C_{5}\right)$, $69.2\left(C_{13}\right), 64.0\left(C_{11}\right), 56.0\left(C_{10}\right), 36.3\left(C_{2}\right), 32.7$ $\left(C_{20}\right), 30.7\left(C_{3}\right), 29.3-29.7\left(C_{15-19}\right), 26.1\left(C_{15}\right), 25.3$ $\left(\mathrm{C}_{12}\right)$.

P5 : $95 \%$ yield, ${ }^{1} \mathrm{H}$ NMR (300 $\left.\mathrm{MHz}, \mathrm{CDCl}_{3}, \delta\right)$ :

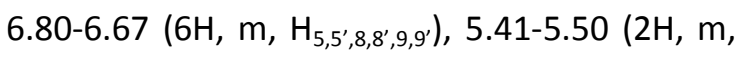
$\left.\mathrm{H}_{18,18^{\prime}}\right), 5.18\left(1 \mathrm{H}, \mathrm{d}, J\right.$ J 3.0, $\left.\mathrm{H}_{11^{\prime}}\right), 5.12(1 \mathrm{H}, \mathrm{q}, J$ J 5.7, $\left.\mathrm{H}_{11}\right), 4.79\left(1 \mathrm{H}, \mathrm{t}, J\right.$ 4.9, $\left.\mathrm{H}_{13^{\prime}}\right), 4.39(1 \mathrm{H}, \mathrm{d}, J$. 4.7, $\left.\mathrm{H}_{13}\right), 3.84\left(6 \mathrm{H}, \mathrm{s}, \mathrm{H}_{10,10^{\prime}}\right), 3.72-4.02(8 \mathrm{H}, \mathrm{m}$,

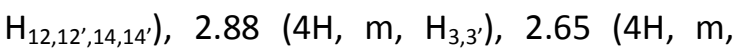

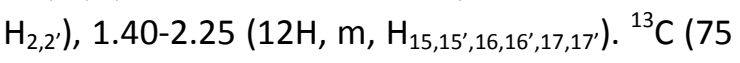
$\left.\mathrm{MHz}, \mathrm{CDCl}_{3}, \delta\right): 172.4\left(\mathrm{C}_{1^{\prime}}\right), 172.1\left(\mathrm{C}_{1}\right), 149.5$ $\left(C_{6,6^{\prime}}\right), 147.3$ and $147.2\left(C_{7,7^{\prime}}\right), 133.0\left(C_{4}\right), 132.8$ $\left(\mathrm{C}_{4^{\prime}}\right), 130.8$ and $130.4\left(\mathrm{C}_{18,18^{\prime}}\right), 120.3\left(\mathrm{C}_{9,9^{\prime}}\right), 113.2$ $\left(C_{8,8^{\prime}}\right), 112.2\left(C_{5,5^{\prime}}\right), 86.0\left(C_{13^{\prime}}\right), 80.9\left(C_{13}\right), 78.1$ $\left(C_{11^{\prime}}\right), 74.0\left(C_{11}\right), 73.5\left(C_{12^{\prime}}\right), 70.5\left(C_{12}\right), 69.1$ $\left(C_{14,14^{\prime}}\right), 56.1\left(C_{10,10^{\prime}}\right), 36.1\left(C_{2^{\prime}}\right), 35.9\left(C_{2}\right), 32.4$ 


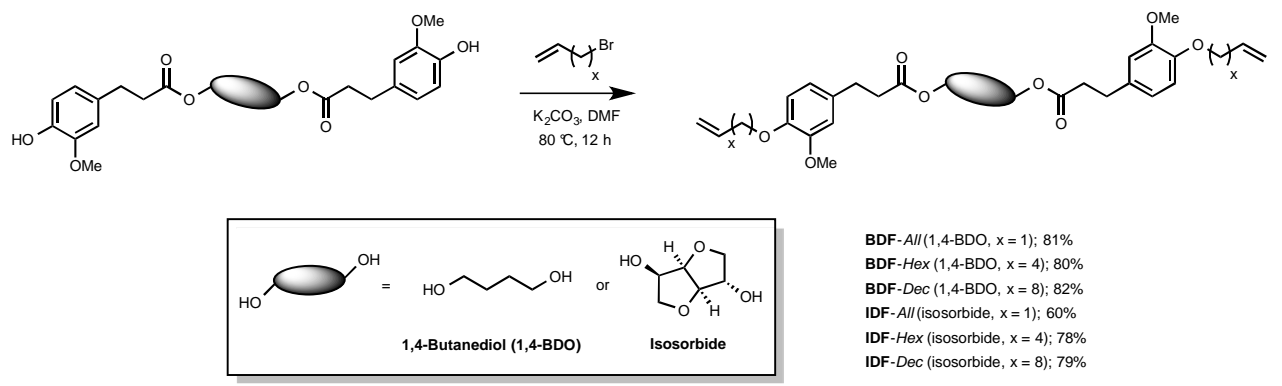

Scheme 2. Synthesis of the six ferulic acid-derived $\alpha, \omega$-diene monomers

$\left(C_{17,17^{\prime}}\right), 30.6\left(C_{3,3^{\prime}}\right), 29.0$ and $28.8\left(C_{15,15^{\prime}}\right), 26.0$ $\left(\mathrm{C}_{16,16^{\prime}}\right)$.

P6 : 94\% yield, ${ }^{1} \mathrm{H}$ NMR (300 $\left.\mathrm{MHz}, \mathrm{CDCl}_{3}, \delta\right)$ : 6.67-6.80 (6H, m, $\left.\mathrm{H}_{5,5^{\prime}, 8,8^{\prime}, 9,9^{\prime}}\right), 5.38\left(2 \mathrm{H}, \mathrm{m}, \mathrm{H}_{22}\right)$, $5.35\left(1 \mathrm{H}, \mathrm{d}, J 2.9, \mathrm{H}_{11}\right), 5.14\left(1 \mathrm{H}, \mathrm{q}, J 5.8, \mathrm{H}_{11}\right)$, $4.79\left(1 \mathrm{H}, \mathrm{t}, J 4.8, \mathrm{H}_{13^{\prime}}\right), 4.39\left(1 \mathrm{H}, \mathrm{d}, J \mathrm{~J} .8, \mathrm{H}_{13}\right)$,

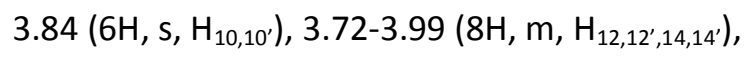
$2.89\left(4 \mathrm{H}, \mathrm{m}, \mathrm{H}_{3,3^{3}}\right), 2.65\left(4 \mathrm{H}, \mathrm{m}, \mathrm{H}_{2,2^{2}}\right), 1.97(4 \mathrm{H}$, $\left.\mathrm{m}, \mathrm{H}_{15,15^{\prime}}\right), 1.82\left(4 \mathrm{H}, \mathrm{m}, \mathrm{H}_{17,17^{\prime}}\right), 1.20-1.50(2 \mathrm{OH}$,

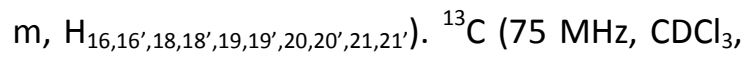
ठ): $172.4\left(C_{1^{\prime}}\right), 172.1\left(C_{1}\right), 149.5\left(C_{6,6^{\prime}}\right), 147.3$ and $147.2\left(C_{7,7^{\prime}}\right), 133.0\left(C_{4}\right), 132.8\left(C_{4^{\prime}}\right), 130.5\left(C_{22}\right)$, $120.3\left(C_{9,9^{\prime}}\right), 113.2\left(C_{8,8^{\prime}}\right), 112.2\left(C_{5,5^{\prime}}\right), 86.0\left(C_{13^{\prime}}\right)$, $80.9\left(C_{13}\right), 78.1\left(C_{11^{\prime}}\right), 74.0\left(C_{11}\right), 73.5\left(C_{12^{\prime}}\right), 70.5$ $\left(C_{12}\right), 69.3\left(C_{14,14^{\prime}}\right), 56.1\left(C_{10,10^{\prime}}\right), 36.1\left(C_{2^{\prime}}\right), 35.9$ $\left(C_{2}\right), \quad 32.7 \quad\left(C_{21,21^{\prime}}\right), \quad 30.6 \quad\left(C_{3,3^{\prime}}\right), \quad 29.3-29.8$ $\left(C_{15,15^{\prime}, 17,17^{\prime}, 18,18^{\prime}, 19,19^{\prime}, 20,20^{\prime}}\right), 26.1\left(C_{16,16^{\prime}}\right)$.

\section{RESULTS AND DISCUSSION}

Syntheses of the ferulic acid-derived $\alpha$, $\omega$-diene monomers

We started our study with the one-step synthesis of the different monomers from IDF and BDF, two bisphenols previously synthesized in our group, ${ }^{8}$ and three bromo-alkenes (3bromoprop-1-ene, 6-bromohex-1-ene et 10bromodec-1-ene) in order to study the impact of both the chain length of the olefin and the rigidity/flexibility of the diester core of the monomer on the polymerizations and the thermal properties of the resulting poly(ester- alkenamer)s. Isosorbide, readily obtained from $\operatorname{corn}^{13}$ was chosen for its robust bicycling structure $^{14}$, potentially providing good thermal and mechanical properties to the resulting polymers. For comparison, 1,4-butanediol, obtained by fermentation of sugars, ${ }^{15}$ was used as a flexible aliphatic segment. Etherification of IDF and BDF was first performed using the procedure previously reported by Meier et al. ${ }^{12 \mathrm{~g}}$ $\left(\mathrm{K}_{2} \mathrm{CO}_{3}, \mathrm{CH}_{3} \mathrm{CN}\right.$, TBAl, reflux, 16 hours) and provided the corresponding $\alpha, \omega$-dienes in 60 $85 \%$ yields. It is noteworthy that similar yields can be obtained without phase-transfer agent (i.e., TBAI) by conducting the etherification in DMF at $80^{\circ} \mathrm{C}$, for 12 hours under nitrogen, in presence of potassium carbonate.

\section{ADMET Polymerization}

With the six ferulic acid-derived $\alpha, \omega$-diene monomers in hand, we proceeded to the study of their behavior in ADMET polymerization. Though it has been reported that ADMET polymerizations are favored when carried out under bulk conditions, ${ }^{16}$ we also performed polymerizations in solvent to verify if this also applied to IDF- and BDF-based $\alpha, \omega$-diene monomers. In the case of ADMET reactions conducted in mass, continuous vacuum (ca. 20 mbar) was applied to guarantee the efficient removal of ethylene and thus shift the equilibrium towards polymerization; in addition, two temperatures (80 and $100{ }^{\circ} \mathrm{C}$ ) have been tested. Dichloromethane, previously reported as a preferred solvent to achieve high 


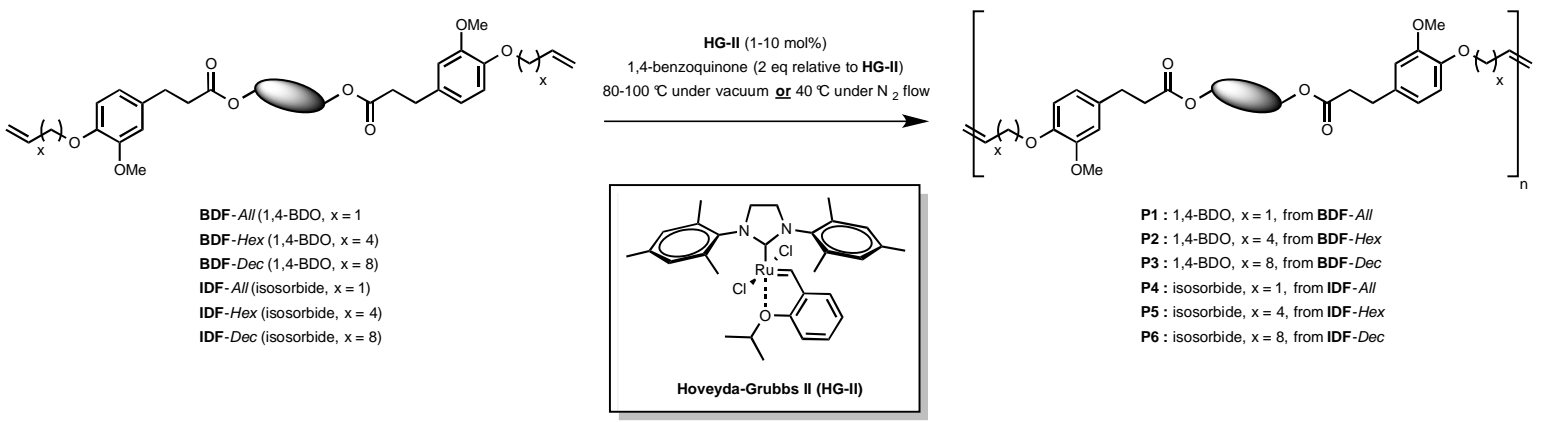

Scheme 3. ADMET polymerization of the six $\alpha, \omega$-diene monomers derived from ferulic acid

polymerization degrees in ADMET, ${ }^{17}$ was used for in solvent polymerizations. In solvent reactions were performed at $40^{\circ} \mathrm{C}$, under a low and steady nitrogen flow to remove ethylene but not dichloromethane. In both reaction conditions, 1,4-benzoquinone ( 2 eq. relative to catalyst) was used to prevent olefin isomerization $\left(2 \%\right.$ mol). ${ }^{18}$ Finally, HoveydaGrubbs second generation catalyst (HG-II), with loadings varying from 1 to $10 \mathrm{~mol} \%$, was the only catalyst tested in this study. Indeed, previous works by Abbas $^{19}$ and Meier ${ }^{12 g}$ have shown that HG-II was the most active towards the cross metathesis of terminal olefins with acrylates and vanillin-based $\alpha, \omega$-dienes, respectively. All ADMET reactions were ran for 4 hours and followed by GPC in order to identify the best conditions (nature of the $\alpha, \omega$-diene monomer, in mass vs. in solvent, temperature, catalyst loading).

\section{Structural analysis}

We first studied the reactivity of the $\alpha, \omega$-diene monomers in solvent and in mass with the following conditions: HG-II $1 \mathrm{~mol} \%, 2$ eq. 1,4benzoquinone, $80^{\circ} \mathrm{C}$ under vacuum (in mass), $\mathrm{DCM}$ at $40{ }^{\circ} \mathrm{C}$ (in solvent), reaction time: 4 hours. In addition to GPC, ${ }^{1} \mathrm{H}$ NMR spectrometry of the resulting crude polymerization mixtures was also performed to not only confirm the poly(ester-alkenamer)s structures but also reveal potential undesired side reactions such as ring-closing metathesis (RCM) or olefin isomerization. Table 1 summarizes the results of these polymerizations. As a representative example, Figure 1-b shows the ${ }^{1} \mathrm{H}$ NMR spectra of BDF-Dec monomers and their corresponding polymers obtained in mass and in solvent, revealing the disappearance of the signals at 4.9-5.1 and 5.7-5.9 ppm corresponding to the terminal olefin protons along with the formation of the internal double bound whose signal is at $5.4 \mathrm{ppm}$. Polymers are obtained from all monomers whatever the reaction conditions, however, in mass, ADMET reactions give higher molecular weights as proven by the residual terminal olefin signals in Figure 1-b and GPC results (Table 1). Indeed, polymerizations performed in solvent (Table 1, Entries 1-4) only lead to short oligomers. Detailed analyses of ${ }^{1} \mathrm{H}$ and ${ }^{13} \mathrm{C}$ NMR spectra show no evidence of isomerization of the double bond (Figure 1; Supporting information).

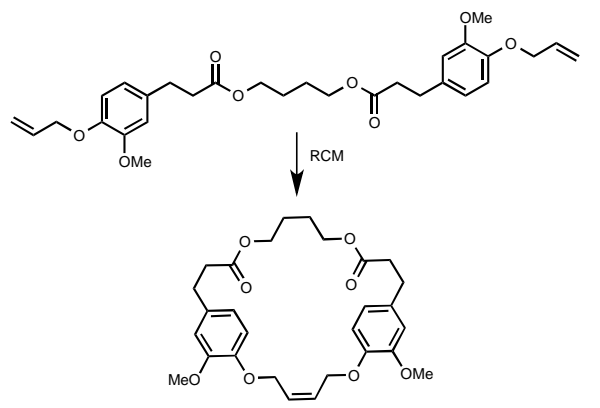

Scheme 4. RCM byproduct obtained during ADMET polymerization of BDF-All

It is also noteworthy that ADMET polymerization of monomers bearing allyl moieties only provides low molecular weight 


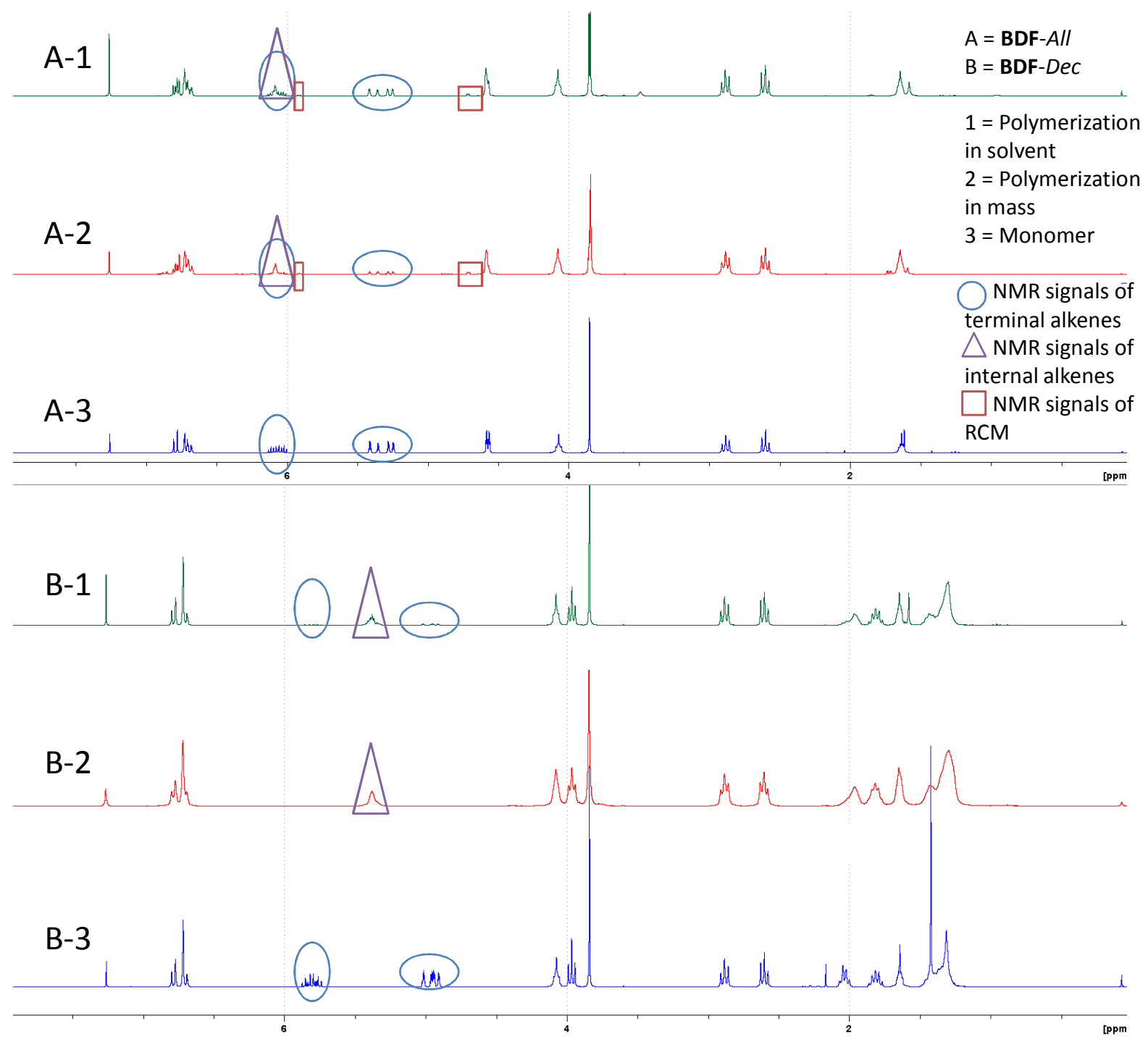

Figure 1. ${ }^{1} \mathrm{H}$ NMR monitoring of ADMET of BDF-All (A) and BDF-Dec (B) in solvent (1) and in mass (2)

polymers (presence of the terminal double bound signals at 5.2-5.5 and 6.0-6.2 ppm). Furthermore, ADMET on BDF-All monomer also leads to the corresponding RCM side-product whose double bound and allylic protons signals at 5.9 and $4.7 \mathrm{ppm}$, respectively, are present in the ${ }^{1} \mathrm{H}$ NMR spectrum (Figure 1-A, Scheme 4). Such RCM product is not observed in the case of IDF-All, probably because of the ring strain of isosorbide and the anti-stereochemistry of its two alcohols that prevent intramolecular metathesis. Allyl-bearing $\alpha, \omega$-diene monomers giving low molecular weights, they are not explored further in the next experiments.

Because in solvent ADMET conditions proved unsuitable for our monomers, only results for poly(ester-alkenamer)s obtained in mass (Table 1, Entries 5-12) are being considered in the following discussion. Whatever the bromo alk1-ene used, higher molecular weights are always obtained with BDF-based $\alpha, \omega$-dienes (P2). Such results could be explained by the higher flexibility of the aliphatic chain of 1,4butanediol compared to the rigid bicyclic 
Table 1. GPC and TGA analytical data of the synthesized polymers via ADMET

\begin{tabular}{|c|c|c|c|c|c|c|c|c|c|}
\hline Entry & ADMET conditions & Monomer & Polymer & $M_{w}{ }^{\mathrm{c}}(\mathrm{kDa})$ & $M_{n}^{\mathrm{c}}(\mathrm{kDa})$ & $D P_{n}{ }^{\mathrm{c}}$ & $D_{\mathrm{M}}^{\mathrm{c}}$ & $T_{d 5 \%}{ }^{\mathrm{d}}\left({ }^{\circ} \mathrm{C}\right)$ & $T_{g}^{\mathrm{e}}\left({ }^{\circ} \mathrm{C}\right)$ \\
\hline 1 & In solvent $^{\mathrm{a}}$ & BDF-Hex & $\mathbf{P 2}$ & 1.6 & 1.4 & 2 & 1.1 & 305.4 & -26.5 \\
\hline 2 & In solvent $\mathrm{t}^{\mathrm{a}}$ & BDF-Dec & $\mathbf{P 3}$ & 5.9 & 5.1 & 8 & 1.2 & 349.8 & -28.4 \\
\hline 3 & In solvent ${ }^{\mathrm{a}}$ & IDF-Hex & P5 & 3.4 & 2.3 & 4 & 1.5 & 305.2 & -7.3 \\
\hline 4 & In solvent ${ }^{\mathrm{a}}$ & IDF-Dec & P6 & 2.0 & 2.0 & 3 & 1.0 & 327.5 & -21.0 \\
\hline 5 & $\operatorname{In~mass~}^{\mathrm{b}}\left(80^{\circ} \mathrm{C}\right)$ & BDF-Hex & $\mathbf{P 2}$ & 26.5 & 20.5 & 34 & 1.3 & 283.3 & -12.1 \\
\hline 6 & In mass ${ }^{\mathrm{b}}\left(80^{\circ} \mathrm{C}\right)$ & BDF-Dec & $\mathbf{P 3}$ & 10.4 & 6.3 & 10 & 2.3 & 370.1 & -21.6 \\
\hline 7 & In mass ${ }^{\mathrm{b}}\left(80^{\circ} \mathrm{C}\right)$ & IDF-Hex & P5 & 12.5 & 5.4 & 8 & 1.6 & 286.8 & -6.2 \\
\hline 8 & In mass ${ }^{\mathrm{b}}\left(80^{\circ} \mathrm{C}\right)$ & IDF-Dec & P6 & 11.8 & 5.9 & 8 & 2.0 & 344.2 & 0.1 \\
\hline 9 & In mass ${ }^{\mathrm{b}}\left(100^{\circ} \mathrm{C}\right)$ & BDF-Hex & $\mathbf{P 2}$ & 42.8 & 25.4 & 42 & 1.7 & 313.9 & -14.9 \\
\hline 10 & In mass ${ }^{\mathrm{b}}\left(100^{\circ} \mathrm{C}\right)$ & BDF-Dec & P3 & 20.5 & 12.3 & 19 & 1.7 & 314.4 & -23.3 \\
\hline 11 & In mass ${ }^{\mathrm{b}}\left(100^{\circ} \mathrm{C}\right)$ & IDF-Hex & P5 & 27.2 & 10.6 & 16 & 2.6 & 291.0 & -8.3 \\
\hline 12 & In mass ${ }^{\mathrm{b}}\left(100^{\circ} \mathrm{C}\right)$ & IDF-Dec & P6 & 21.6 & 9.9 & 14 & 2.2 & 333.6 & -7.8 \\
\hline
\end{tabular}

${ }^{a}$ HG-II 1 mol\%, 1,4-benzoquinone (2 mol\%),DCM (2 M), $40{ }^{\circ} \mathrm{C}, 4$ hours; ${ }^{b}$ HG-II 1 mol\%, 1,4-benzoquinone (2 mol\%), vacuum, 4 hours; ${ }^{\mathrm{C}} \mathrm{GPC}$ data of crude polymers; ${ }^{\mathrm{d}}$ TGA data under nitrogen at $60 \mathrm{~mL} \cdot \mathrm{min}^{-1} ;{ }^{\mathrm{e}} \mathrm{DSC}$ data recorded at $10^{\circ} \mathrm{C} \cdot \mathrm{min}^{-1}$, value determined at the $2^{\text {nd }}$ heating scan

structure of isosorbide and the resulting lower viscosity of BDF-based monomers. However, interestingly, replacing hex-1-ene by dec-1-ene (Table 1, Entries 6, 8, 10 and 12) does not provide higher molecular weights. Furthermore, while molecular weights of IDF-derived poly(ester-alkenamer)s (P5 vs. P6) remain unchanged or increase by only $14 \%$ when dec-1ene was replaced by hex-1-ene (Table 1, Entries 7-8 and 11-12), those of BDF-derived polymers (P2 vs. P3) significantly increase by $121-140 \%$ (Table 1, Entries 5-6 and 9-10). As shown in Table 1, heating the reaction mixture from 80 to $100{ }^{\circ} \mathrm{C}$ significantly improves the molecular weights by lowering the viscosity of the reaction medium and thus facilitating both ethylene removal and stirring. The IDF-based monomers are the ones that benefit most from this rise in temperature resulting in a molecular weight increase of ca. $100.5 \%$ for IDF (82.5\% for BDF).
Finally, as expected, increasing the catalyst loading above 1 mol\% resulted in decreasing molecular weights. A representative example with BDF-Hex is shown in Figure 2. It is noteworthy, that the use of lower catalyst loadings (e.g., $0.1-0.5 \mathrm{~mol} \%$ ) or longer reactions times (8 hours) did not lead to higher molecular weight.

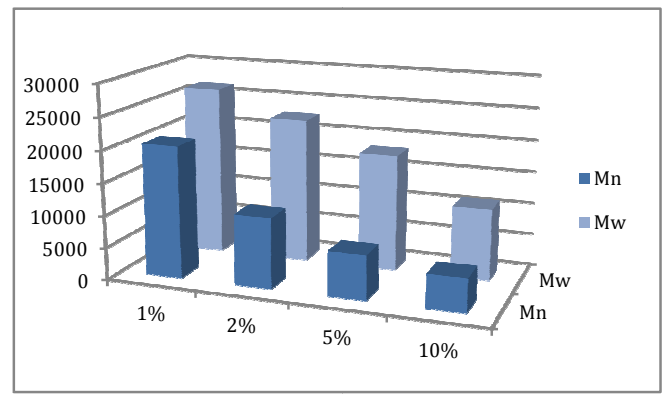

Figure 2. Influence of loading catalyst in BDFHex polymerization 
<smiles>C=CNc1ccc(COC(=O)CCCCC(=O)OCc2ccc(ON=CC)c(OC)c2)cc1OC</smiles>
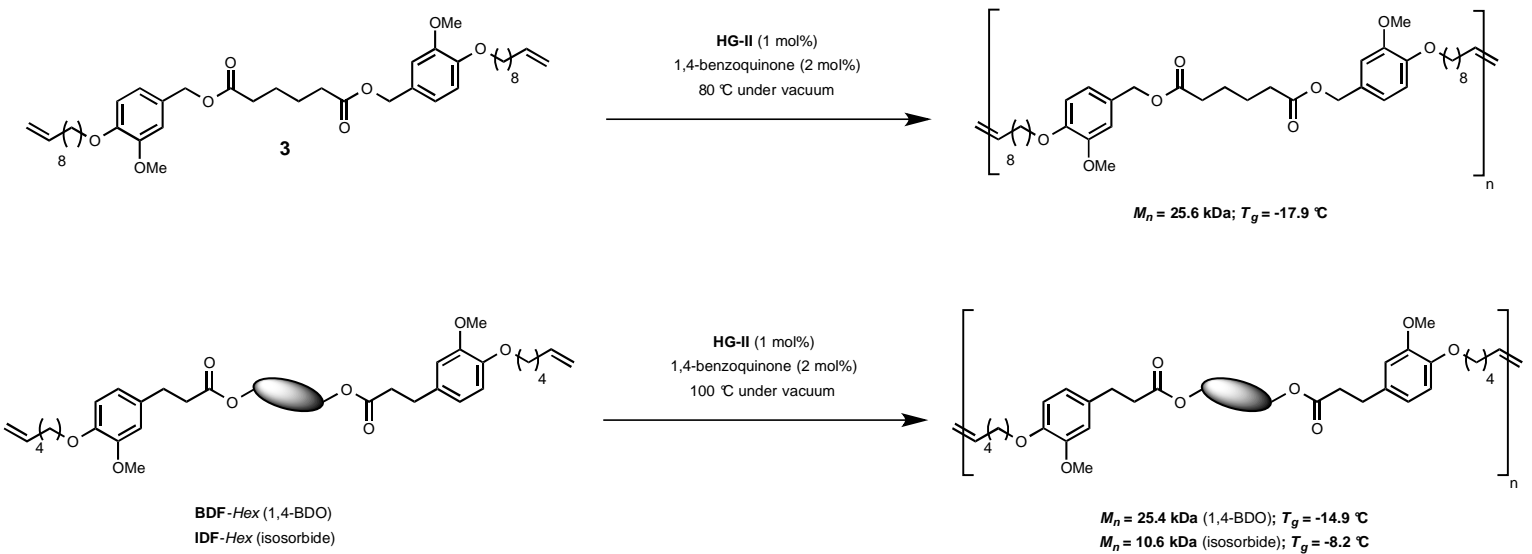

Scheme 5. Ferulic acid- and vanillin-derived ${ }^{12 g}$ poly(ester-alkenamer)s via ADMET

On the basis of the results reported in Table 1 and Figure 2, it was concluded that in mass reaction conducted with $1 \mathrm{~mol} \% \mathrm{HG}-\mathrm{II}$, at $100{ }^{\circ} \mathrm{C}$ for 4 hours was the best procedure for ADMET polymerization for all monomers. In such conditions, BDF- and IDF-based poly(esteralkenamer)s (P2/P3 and P5/P6) were obtained with number average molecular weight $\left(M_{n}\right)$ in the range of 12.3-25.4 and 9.9-10.6 kDa, respectively. In terms of reactivity, BDF-Hex $\alpha, \omega$ ? diene thus proves more reactive than monomer 2 previously reported by Meier and co-workers $^{12 a}$ and as efficient as their recently published vanillin-derived $\alpha, \omega$-diene 3 which gives similar molecular weights when submitted to ADMET polymerization with HG-II (Scheme 5). ${ }^{12 \mathrm{~g}}$

\section{Thermal properties}

The thermal properties of monomers and polymers described in Table 1 have been investigated by thermogravimetric analysis (TGA) and differential scanning calorimetry (DSC).

\begin{tabular}{ccccc}
\hline Monomer & BDF-Hex & BDF-Dec & IDF-Hex & IDF-Dec \\
\hline$T_{d 5 \%}\left({ }^{\circ} \mathrm{C}\right)$ & 302.5 & 327.3 & 335.3 & 348.5 \\
\hline
\end{tabular}

Table 2. Thermostability of BDF- and IDF-based monomers
TGA analyses of the monomers revealed a thermostability in the range of $302-349{ }^{\circ} \mathrm{C}$ (Table 2) but also that the nature of the diester significantly impacts the thermostability of the monomers. IDF-based monomers prove more stable than the ones deriving from BDF. Furthermore, the nature of the alkene impacts the degradation temperature $\left(T_{d} 5 \%\right)$, indeed replacing hex-1-ene by dec-1-ene in BDF- and IDF-based monomers strongly increases their thermostability by 24.8 and $13.2{ }^{\circ} \mathrm{C}$ respectively.

Thermal analyses of polymers P2-P6 showed significant differences in $T_{d} \quad 5 \%$ and glass transition temperature $\left(T_{g}\right)$ depending on the nature of the internal diester (1,4-butanediol vs. isosorbide) and the alkene length ( $C_{6}$ vs. $C_{10}$ ). Aromatic in nature, all poly(ester-alkenamer)s exhibit a thermostability in the range of 283$370{ }^{\circ} \mathrm{C}$, the dec-1-ene based ones being the most stable (Table 1, Entries 6, 8, 10 and 12). The nature of the internal diester, however, has a relatively low impact on the degradation temperature (Table 1, Entries 9-12). Similarly to what was observed with the previously reported copolyesters ${ }^{9}$ and poly(esterurethane) $\mathrm{s}^{10}$ derived from these ferulic acidbased bisphenols, the rigid bicyclic structure of isosorbide also greatly increases the $T_{g}$ of poly(ester-alkenamer)s (P5 and P6) compared to their BDF-based counterparts (P2 and P3). 
Varying the nature of the $\alpha, \omega$-diene monomers thus provides $T_{g}$ in the range of -28.4 to $0.1{ }^{\circ} \mathrm{C}$. From a strictly thermal properties perspective, BDF-Dec based poly(ester-alkenamer) (P3) could thus be seen as a renewable alternative to polyethylene (PE). ${ }^{20}$ Finally, it is noteworthy that all obtained polymers are amorphous and do not show melting points $\left(T_{m}\right)$ on DSC.

\section{CONCLUSIONS}

In summary, ferulic-acid based $\alpha, \omega$-diene monomers have been obtained in good yields through a chemo-enzymatic synthetic pathway and were successfully polymerized via ADMET in presence of second generation HoveydaGrubbs catalyst. In mass polymerization resulted in poly(ester-alkenamer)s with $M_{n}$ as high as $25.4 \mathrm{kDa}$ in the case of 1,4-butanediolderived monomers. Thermal analyses through TGA and DSC demonstrated that such polymers are thermostable up to $283-370{ }^{\circ} \mathrm{C}$ and, more importantly, that their $T_{g}$ can be easily tuned by playing with both the nature of the internal diester and the alkene length of the $\alpha, \omega$-diene monomers. Prepared from renewable feedstocks (ferulic acid, 1,4-butanediol, isosorbide), these polymers could be envisaged as sustainable substitutes to conventional petro-based polyesters.

\section{ACKNOWLEDGEMENTS}

The authors are grateful to the Region Champagne-Ardenne, the Conseil Général de la Marne and Reims Métropole for their financial support.

\section{REFERENCES AND NOTES}

1. a) J. J. Bozell, J. O. Hoberg, et al. In Green Chemistry - Frontiers in Benign Chemical Synthesis and Processes, Oxford University, 1998, p. 27-45; b) C. Okkerse, H. van Bekkum, Green Chem. 1999, 1, 107; c) D. L. Klass In Biomass for Renewable Energy, Fuels, and Chemicals - Academic Press, 1998, p. 91-157, p. 495-542
2. L. Mialon, A. G. Pemba and S. A. Miller, Green Chem. 2010, 12, 1704

3. J. Palacios, C. Perez, New Polym. Mat. 1990, 2, 167

4. H. G. Elias, J. A. Palacios, Makromol. Chem. 1985, 5, 1027

5. a) T. Kaneko, M. Matsuaki, T. T. Hang and M. Akashi, Macromol. Rapid Commun. 2004, 25, 673-677; b) Y. Tanaka, T. Tanabe, Y. Shimura, A. Okada, J. Polym. Sci. B Polym. Lett. Ed. 1975, 13, 235; c) B. Sapich and J. Stumpe, Macromolecules 1998, 31, 1016 ; d) J. Stumpe, A. Ziegler, Macromolecules 1995, 28, 5306; e) X. Jin, C. Carfagna, L. Nicolais, R. Lanzetta, Macromolecules 1995, 28, 4785; f) S. Wang, D. Kaneko, K. Kan, X. Jin, T. Kaneko, Pure Appl. Chem. 2012, 12, 2559; g) N. Hatanaka, H. Okawa, KR Patent 2013006318; 2013; h) Q. Zhao, W. Wu, Polymer 2009, 4, 998

6. a) J. Du, Y. Fang, Y. Zheng Polymer 2007, 19, 5541; b) M. A. Ouimet, J. Griffin, A. L. Carbone-Howell, L. Ashley, W.-H. Wu, N. D. Stebbins, R. Di, K. E. Uhrich, Biomacromolecules 2013, 14(3), 854

7. a) M. Quentin, V. Allasia, A. Pegard, F. Allais, P.-H. Ducrot, B. Favery, C. Levis, S. Martinet, C. Masur, M. Ponchet, D. Roby, L. Schlaich, L. Jouanin, H. Keller, PLoS Pathog. 2009, 5(1) doi: 10.1371/journal.ppat.1000264; b) F. Allais, M. Aouhansou, A. Majira, P.-H. Ducrot, Synthesis 2010, 16, 2787; c) F. Allais, P.-H. Ducrot, Synthesis 2010, 16, 1649; d) L. M. M. Mouterde, A. L. Flourat, M. M. M. Cannet, P.-H Ducrot, F. Allais, Eur. J. Org. Chem. 2013, 1, 173; e) B. Cottyn, A. Kollman, P. Waffo Teguo, P.-H. Ducrot, Chem. Eur. J. 2011, 17, 7282; f) J. C. Dean, R. Kusaka, P. S. Walsh, F. Allais, T. S. Zwier, J. Am. Chem. Soc. 2014, 136, 14780

8. F. Pion, A. F. Reano, P.-H. Ducrot and F. Allais, RSC Adv. 2013, 3, 8988

9. F. Pion, P.-H. Ducrot and F. Allais, Macromol. Chem. Phys. 2014, 215, 431

10. M. Z. Oulame, F. Pion, P.-H. Ducrot, F. Allais Eur. Polym. J. 2014, accepted 
11. H. Mutlu, L. M. de Espinosa, M. A. R. Meier, Chem. Soc. Rev. 2011, 40, 1404

12. a) O. Kreye, T. Tóth, M. A. R. Meier, Eur. Polym. J. 2011, 47, 1804; b) H. Mutlu, M. A. R. Meier, Macromol. Chem. Phys. 2009, 210, 1019; c) L. M. de Espinosa, M. A. R. Meier, J. C. Ronda, M.Galià, V. Cadiz, J. Polym. Sci. Part A: Polym. Chem. 2010, 48, 1649; d) T. W. Baughman; K. B. Wagener, Adv. Polym. Sci. 2005, 176, 1; e) O. Türünç, L. M. de Espinosa, M. A. R. Meier, Macromol. Rapid. Commun. 2011, 32, 1357; f) S. Günther, P. Lamprecht, G. A. Luinstra, Macromol. Symp. 2010, 293, 15; g) M. Firdaus, M. A. R. Meier, Eur. Polym. J. 2013, 49, 156

13. T. Carde, DOE/GO-102001-1461; New Continuous Isosorbide Production From Sorbitol: Office of Industrial Technologies (OIT) Agriculture Project Fact Sheet, 2012

14. J. Adelman, L. F. Charbonneau and S. Ung 2003. US Patent $6,656,577,2003$, USA
15. a) S. Kobayashi, Macromol. Rapid. Commun. 2009, 30, 237; b) H. Yim, R. Haselbeck, W. Niu, C. Pujol-Baxley, A. Burgard, J. Boldt, J. Khandurina, J. D. Trawick, R. E. Osterhout, R. Stephen, J. Estadilla, S. Teisan, H. B. Schreyer, S. Andrae, T. H. Yang, S. Y. Lee, M. J. Burk, S. Van Dien, Nat. Chem. Biol. 2011, 7, 445; c) S. Schauhoff, W. Schmidt, Chem. Fibers Int. 1996, 46, 263; d) D. R. Kelsey US Patent 6,093,786; 2000

16. M. D. Watson, K. B. Wagener, Macromolecules 2000, 33, 8963

17. M. D. Schulz, K. B. Wagener, ACS Macro Lett. 2012, 1, 449

18. S. H. Hong, D. P. Sanders, C. W. Lee, R. H. Grubb, J. Am. Chem. Soc. 2005, 31, 368

19. M. Abbas, C. Slugovc, Tetrahedron Lett. 2011, 52, 2560

20. U. Gaur, B. Wunderlich, Macromolecules $1980,13,445$ 
